\title{
Calculation of Flight Data Fusion Coefficients Using Newton-Raphson Iterations
}

https://doi.org/10.31713/MCIT.2020.20

\author{
Elkhan N. Sabziev \\ Institute of Control Systems \\ Azerbaijan National Academy of Science \\ Baku, Azerbaijan \\ elkhan.sabziev@gmail.com
}

\begin{abstract}
The problem of plotting the flight path of an aircraft based on flight data containing numerous measurement errors is investigated. A theoretical (continuous) model of the flight data fusion problem is proposed in the form of a boundary value problem for a system of differential equations with unknown coefficients. The application of the Newton-Raphson iteration method for calculating the sought-for coefficients is described.
\end{abstract}

Keywords - mathematical model, data fusion, aircraft, black box, differential equations, iteration method.

\section{INTRODUCTION}

When first introduced in aviation, flight data recorder systems were an important step in the development of aviation. A flight data recorder is primarily used for studying the behavior of the aircraft after flight to establish what happened in the event of an accident. The data recorded for this purpose include yaw, pitch, and roll angles, which determine the aerial orientation of the aircraft, loads expressing forces that determine the movement of the aircraft, absolute speed, readings of the barometric altimeter and other parameters. Determining the current location of the aircraft based on flight data received from measuring instruments is also one of the key problems in military aviation. The problem of determining the location and direction of the aircraft during flight, as a rule, is due to the difficulties of determining the aerial orientation the pilot experiences when performing combat tasks in complicated operating conditions.

Nowadays, aircrafts are equipped with more advanced models of measuring instruments. These devices, the first models of which appeared in the first half of the twentieth century and which have been known in science as "black box", include gyroscopes, accelerometers, barometric altimeters, speed meters and other devices operating on various laws of fundamental physics.

The use of the black box has traditionally been aimed at solving the following problems: - preventing future possible adverse situations by analyzing the causes of various flight incidents; - assessing the technical condition of various instruments and equipment of the aircraft; - assessing pilot's skills in performing flight tasks. To solve these problems, mathematical models of devices based on the physical principle and numerous software systems used in various fields of the aviation industry have been developed.

As a result of intensive development of information technology, GPS systems are now considered navigation systems for everyday use. However, it is generally known that it is impossible to establish the degree of adequacy of performance for a military mission by means of GPS. One of the reasons is that GPS signals come in intermittently, coordinates are determined with errors and, most importantly, GPS signals in areas of planned military operations cannot be received by appropriate devices as a result of noise pollution. Therefore, the problem of determining a military aircraft's location in the air and its orientation relative to the Earth on the basis of the data recorded in its black box arises.

It should be noted that the indicators recorded by navigation devices in actual flight contain various errors and distortions, and many problems arise during their processing. However, open sources generally do not give a detailed description of these problems, and information on the relevant studies is not disclosed for obvious reasons. There are also some papers by Azerbaijani researchers on the problems of flight data processing worth mentioning: [1 - 5].

Therefore, in this paper, we investigate the problem of restoring the flight path of an aircraft after flight, which is caused by errors in the black box data, proposing a data fusion method to solve it.

\section{THE ESSENCE OF THE PROBLEM}

Based to the operating principle, navigation devices used in airplanes can be grouped into two categories: "strapdown" and "platform-type" systems. The first category includes, for instance, a TEST-UZ type navigation device [6]. This type of devices is used in An-24, MiG-AT, SU-24, SU-27 and other 


\section{Modeling, control and information technologies -2020}

aircrafts. An example of the second category of navigation devices is the IKV-1 navigation system [7]. This type of devices is used, for instance, in aircrafts like Yak-38, MIG-23B, MIG27, SU-17.

The aforementioned navigation devices measure such aircraft parameters as loads (accelerometer), yaw, pitch and roll angles (gyroscope), velocity (Pitot tube), flight altitude (barometric altimeter). Depending on the operating principle of the device, these quantities are recorded in the coordinate system relative to the Earth. For clarity, we shall briefly explain the quantities used in this paper.

Let us introduce a rectangular inertial coordinate system $O x y z$, fixing it to the point of aircraft's liftoff from the runway. The coordinate axes are directed, as is customary in the aviation literature, as follows: the $O x$ and $O z$ axes located on a horizontal plane are directed along the adopted references, and the $O y$ axis is directed perpendicular upward from the earth's surface.

$\psi$ - the yaw angle is the angle between the aircraft's flight direction and the direction selected as the reference. As a rule, the northern direction of the horizon, or, in some cases, the eastern direction, is selected as the reference. Suppose the direction of the $O x$ axis is taken as the reference direction.

$\boldsymbol{n}=\left(n_{x}, n_{y}, n_{z}\right)-$ by the load we understand the ratio of the sum of all aerodynamic forces acting on the aircraft, except gravity, and engine thrust to gravity. We can write the components of the load vector relative to the Earth by applying transformation formulas written on the basis of orientation angles. This allows calculating the acceleration created by the forces acting on the aircraft in the coordinate system relative to the Earth.

As noted above, numerous errors occur when velocity, loads, orientation angles and other important flight parameters of the aircraft are measured, digitized and recorded in the black box. Therefore, the restoration of the flight path with adequate accuracy, directly using these indicators after flight, becomes a challenging task.

Ideally, the first and second derivatives of the actual flight path $\boldsymbol{s}(t)=(x(t), y(t), z(t))$, the equation of which is written with respect to the inertial system, should coincide with the velocity $\boldsymbol{v}(t)$ and acceleration $\boldsymbol{a}(t)$, respectively, obtained from the primary processing of black box data. However, we actually observe $\boldsymbol{s}^{\prime}(t) \neq \boldsymbol{v}(t)$ and $\boldsymbol{s}^{\prime \prime}(t) \neq \boldsymbol{a}(t)$, and the readings $h(t)$ of the barometric altimeter differ from the real function $y(t)$.

In this study, a flight data fusion hypothesis is put forward, suggesting that there is a linear combination of indicators recovered from flight data such that its value coincides with the corresponding combination recorded for the actual flight path.

Thus, it is assumed that the movement of an aircraft (airplane) in flight is described by a differential equation with constant coefficients that depend mainly on its weight and aerodynamic properties.

Therefore, if we neglect the aircraft mass variation, taking into account its smallness relative to the weight of the entire machine, then the motion equation will be simplified substantially. When modeling the motion, we shall not go into the nuances of aerodynamics, assuming that its effect is indirectly reflected in the fusion coefficients. The working hypothesis is based on the following mathematical considerations:

1) When setting up a linear differential equation with respect to a prescribed function $v=v(t), t \in[0, T]$, using any reasonable differential operation $L$, the right-hand side of the equation will be the function $F=L v$. The equation for the sought-for function $u=u(t)$ is written as $L u=F$.

2) If the initial data coincide $\left.B u\right|_{t=0}=\left.B v\right|_{t=0}$, the solution $u(t)$ of the equation $L u=F$ coincides with the function generating the right-hand side: $u(t)=v(t), t \in[0, T]$.

3) If different approximations of $v$ are used in the rule of generation $F=L v$, then the value of $u(t)$ at the point $t=T$ can coincide with the value of $v(T)$ for some specially selected coefficients of the operation $F$.

\section{MATHEMATICAL MODEL OF THE FUSION PROBLEM}

Mathematically, the essence of the fusion method is determining the vector of the aircraft's location $\boldsymbol{s}=$ $(x(t), y(t), z(t))$ as a solution to the following system of linear differential equations

$$
\left\{\begin{array}{c}
x^{\prime \prime}-k_{1} x^{\prime}=a_{x}-k_{1} \sqrt{v^{2}-\left(y^{\prime}\right)^{2}} \cos \psi ; \\
y^{\prime \prime}-k_{2} y=a_{y}-k_{2} h \\
z^{\prime \prime}-k_{3} z^{\prime}=a_{z}-k_{3} \sqrt{v^{2}-\left(y^{\prime}\right)^{2}} \sin \psi ; \\
t \in(0, T)
\end{array}\right.
$$

with initial conditions at $t=0$

$$
\left\{\begin{array}{c}
x(0)=0, y(0)=0, z(0)=0 ; \\
x^{\prime}(0)=x_{0}, y^{\prime}(0)=y_{0}, z^{\prime}(0)=z_{0}
\end{array}\right.
$$

where $a_{x}, a_{y}, a_{z}$ are longitudinal, vertical and lateral accelerations, respectively; $h$ is the estimate of baro-inertial altitude; $\psi$ is the yaw angle; $v=v(t)$ is the actual velocity; $k_{1}$, $k_{2}, k_{3}$ are the fusion filter tuning coefficients; $T$ is flight duration, $\left(x_{0}, y_{0}, z_{0}\right)$ is aircraft's velocity at the moment of liftoff from the runway.

In contrast to [5], the derivative of $h$ is not included in system (1), since numerical experiments show that taking such a term into account does not lead to a significant improvement in the results.

The structure of problem (1)-(2) is such that it can be split into separate tasks and solved successively: first with respect to $y$, then with respect to $x$ and $z$. In this case, an explicit dependence of the functions $y, x$ and $z$ on the fusion parameters $k_{1}, k_{2}, k_{3}$ can be represented as follows:

$$
x=x\left(k_{1}, t\right) ; y=y\left(k_{2}, t\right) ; z=z\left(k_{3}, t\right)
$$

The coefficients $k_{1}, k_{2}, k_{3}$ will be determined from the conditions of minimization of residuals

$$
\left|x\left(k_{1}, T\right)-x_{T}\right|,\left|y\left(k_{2}, T\right)-y_{T}\right|,\left|z\left(k_{3}, T\right)-z_{T}\right|,
$$

where $\left(x_{T}, y_{T}, z_{T}\right)$ is the relative coordinates of the arrival airfield. 


\section{Modeling, control and information technologies - 2020}

\section{SOLUTION}

First, the approximate value of the coefficient $k_{2}$ is determined. Assuming $k_{2}>0$, denote $\alpha=\sqrt{k_{2}}$.

From (1)-(2), the explicit representation of the function $y\left(k_{2}, t\right)$ can be written as follows:

$$
\begin{gathered}
y\left(k_{2}, t\right)=\frac{y_{0}\left(e^{\alpha t}-e^{-\alpha t}\right)}{2 \alpha}+ \\
\int_{0}^{t} \frac{e^{\alpha(t-\tau)}-e^{-\alpha(t-\tau)}}{2 \alpha}\left(a_{y}(\tau)-\alpha^{2} h(\tau)\right) d \tau
\end{gathered}
$$

From the condition of residual minimization for $y\left(k_{2}, T\right)$, we have

$$
\begin{gathered}
\frac{y_{0}\left(e^{\alpha T}-e^{-\alpha T}\right)}{\alpha}+ \\
\int_{0}^{T} \frac{e^{\alpha(T-\tau)}-e^{-\alpha(T-\tau)}}{2 \alpha}\left(a_{y}(\tau)-\alpha^{2} h(\tau)\right) d \tau-y_{T}=0 .
\end{gathered}
$$

To find $\alpha$, we apply the Newton-Raphson iteration method [8, P.172], indicating the iteration numbers with a superscript: $\alpha^{(j)}, j=0,1,2, \ldots$.

Denote the left-hand side of (4) by $F(\alpha)$. The computation will look as follows:

$$
F\left(\alpha^{(j+1)}\right)=F\left(\alpha^{(j)}\right)+\frac{\partial F\left(\alpha^{(j)}\right)}{\partial \alpha} \Delta \alpha_{j}=0,
$$

where $\alpha^{(j+1)}=\alpha^{(j)}+\Delta \alpha_{j}$.

We take, for instance, $\alpha^{(0)}=1$ as the initial iteration. Thus,

$$
\begin{gathered}
F\left(\alpha^{(j)}\right)=\frac{y_{0}\left(e^{\alpha^{(j)}}-e^{-\alpha^{(j)}}\right)}{2 \alpha^{(j)}}+ \\
\int_{0}^{T} \frac{e^{\alpha^{(j)}(T-\tau)}-e^{-\alpha^{(j)}(T-\tau)}}{2 \alpha^{(j)}}\left(a_{y}(\tau)-\left(\alpha^{(j)}\right)^{2} h(\tau)\right) d \tau-y_{T}, \\
\frac{\partial F\left(\alpha^{(j)}\right)}{\partial \alpha}= \\
\frac{y_{0}}{2\left(\alpha^{(j)}\right)^{2}}\left(e^{\alpha^{(j)} T}\left(T \alpha^{(j)}-1\right)+e^{-\alpha^{(j)} T}\left(T \alpha^{(j)}+1\right)\right)+ \\
\int_{0}^{T}\left[\frac{e^{\alpha^{(j)}(T-\tau)}\left((T-\tau) \alpha^{(j)}-1\right)+e^{-\alpha^{(j)}(T-\tau)}\left((T-\tau) \alpha^{(j)}+1\right)}{2\left(\alpha^{(j)}\right)^{2}} \times\right. \\
\left.\left(a_{y}(\tau)-\left(\alpha^{(j)}\right)^{2} h(\tau)\right)-\left(e^{\alpha^{(j)}(T-\tau)}-e^{-\alpha^{(j)}(T-\tau)}\right) h(\tau)\right] d \tau .
\end{gathered}
$$

Thus, according to $(5)$, the function $y\left(k_{2}, t\right)$ is recovered.

Now, from (1)-(2), we can write the explicit form of the function $x$ :

$$
x\left(k_{1}, t\right)=\frac{x_{0}\left(e^{k_{1} t}-1\right)}{k_{1}}+\int_{0}^{t} \frac{e^{k_{1}(t-\tau)}-1}{k_{1}}\left(a_{x}(\tau)-k_{1} v_{x}(\tau)\right) d \tau
$$

where $v_{x}(t) \equiv \sqrt{v^{2}(t)-\left(y^{\prime}\left(k_{2}, t\right)\right)^{2}} \cos \psi(t)$.

From the condition of residual for the function $x\left(k_{1}, T\right)$, we have

$$
\frac{x_{0}\left(e^{k_{1} T}-1\right)}{k_{1}}+\int_{0}^{T} \frac{e^{k_{1}(T-\tau)}-1}{k_{1}}\left(a_{x}(\tau)-k_{1} v_{x}(\tau)\right) d \tau-x_{T}=0
$$

To find $k_{1}$, we also apply the Newton-Raphson iteration method, indicating the iteration numbers with a superscript: $k_{1}^{(j)}, j=0,1,2, \ldots$

Denote the left-hand side of (5) by $F\left(k_{1}\right)$. The computation will look as follows:

$$
F\left(k_{1}^{(j+1)}\right)=F\left(k_{1}^{(j)}\right)+\frac{\partial F\left(k_{1}^{(j)}\right)}{\partial k_{1}} \Delta k_{1_{j}}=0 .
$$

where $k_{1}^{(j+1)}=k_{1}^{(j)}+\Delta k_{1_{j}}$. We take $k_{1}^{(0)}=1$ as the initial iteration. Thus,

$$
\begin{gathered}
F\left(k_{1}^{(j)}\right)=\frac{x_{0}\left(e^{k_{1}^{(j)} T}-1\right)}{k_{1}^{(j)}}+ \\
\int_{0}^{T} \frac{e^{k_{1}^{(j)}(T-\tau)}-1}{k_{1}^{(j)}}\left(a_{x}(\tau)-k_{1}^{(j)} v_{x}(\tau)\right) d \tau-x_{T}, \\
\frac{\partial F\left(k_{1}^{(j)}\right)}{\partial k_{1}}=x_{0} \frac{e^{k_{1}^{(j)} T}\left(k_{1}^{(j)} T-1\right)+1}{\left(k_{1}^{(j)}\right)^{2}}+ \\
\int_{0}^{T} \frac{e^{k_{1}^{(j)}(T-\tau)}\left(a_{x}(\tau)\left(k_{1}^{(j)}(T-\tau)-1\right)-\left(k_{1}^{(j)}\right)^{2}(T-\tau) v_{x}(\tau)\right)+a_{x}(\tau)}{\left(k_{1}^{(j)}\right)^{2}} d \tau .
\end{gathered}
$$

Solving equation (6) numerically, we find the coefficient $k_{1}$, therefore, the function $x\left(k_{1}, t\right)$ is recovered.

Finally, from (1)-(2), we can write the explicit form of the function $z$ :

$$
z\left(k_{3}, t\right)=\frac{z_{0}\left(e^{k_{3} t}-1\right)}{k_{3}}+\int_{0}^{t} \frac{e^{k_{3}(t-\tau)}-1}{k_{3}}\left(a_{z}(\tau)-k_{3} v_{z}(\tau)\right) d \tau
$$

where $v_{z}(t) \equiv \sqrt{v^{2}(t)-\left(y^{\prime}\left(k_{2}, t\right)\right)^{2}} \sin \psi(t)$.

Also from the condition of residual for the function $z\left(k_{3}, T\right)$, we have

$$
\frac{z_{0}\left(e^{k_{3} T}-1\right)}{k_{3}}+\int_{0}^{T} \frac{e^{k_{3}(T-\tau)}-1}{k_{3}}\left(a_{z}(\tau)-k_{3} v_{z}(\tau)\right) d \tau-z_{T}=0
$$

As in the previous cases, to find $k_{3}$, we again apply the Newton-Raphson iteration method, indicating the iteration numbers with a superscript: $k_{3}^{(j)}, j=0,1,2, \ldots$

Denote the left-hand side of $(5)$ by $F\left(k_{3}\right)$. The computation will look as follows:

$$
F\left(k_{3}^{(j+1)}\right)=F\left(k_{3}^{(j)}\right)+\frac{\partial F\left(k_{3}^{(j)}\right)}{\partial k_{3}} \Delta k_{3 j}=0
$$

where $k_{3}^{(j+1)}=k_{3}^{(j)}+\Delta k_{3 j}$.

We take $k_{3}^{(0)}=1$ as the initial iteration. Then

$$
\begin{gathered}
F\left(k_{3}^{(j)}\right)=\frac{z_{0}\left(e^{k_{3}^{(j)} T}-1\right)}{k_{3}^{(j)}}+ \\
\int_{0}^{T} \frac{e^{k_{3}^{(j)}(T-\tau)}-1}{k_{3}^{(j)}}\left(a_{z}(\tau)-k_{3}^{(j)} v_{z}(\tau)\right) d \tau-z_{T}
\end{gathered}
$$


Modeling, control and information technologies -2020

$$
\begin{gathered}
\frac{\partial F\left(k_{3}^{(j)}\right)}{\partial k_{3}}=z_{0} \frac{e^{k_{3}^{(j)} T}\left(k_{3}^{(j)} T-1\right)+1}{\left(k_{3}^{(j)}\right)^{2}}+ \\
\int_{0}^{T} \frac{e^{k_{3}^{(j)}(T-\tau)}\left(a_{z}(\tau)\left(k_{3}^{(j)}(T-\tau)-1\right)-\left(k_{3}^{(j)}\right)^{2}(T-\tau) v_{z}(\tau)\right)+a_{z}(\tau)}{\left(k_{3}^{(j)}\right)^{2}} d \tau .
\end{gathered}
$$

Solving equation (7) numerically, we find the coefficient $k_{3}$, therefore, the function $z\left(k_{3}, t\right)$ is recovered.

\section{CONCLUSION}

We put forward the idea of fusing flight data, representing the flight path equations in the form of their linear combination. An iteration algorithm is proposed for calculating the coefficients of these equations, which ideally coincide with an arbitrarily selected initial approximation.

This study is theoretical in nature. As we know, flight data is measured at discrete instants of time and recorded in the black box. Therefore, for the practical implementation of the method, its discrete analogue should be developed and applied to process large amounts of flight data of each aircraft.

It is assumed that the combined coefficients may be different for each specific aircraft, and the mean values of the coefficients calculated for different flights of that aircraft can be used for data fusion.

\section{REFERENCES}

[1] Alguliyev R.M., Orujov G.G., Azizov R.A., Azizov Y.A. Development of a system for operational processing of flight information // Elmi Məcmuələr (National Aviation Academy journal), - 2005, Vol.7, No 2, p. 58-70. (in Russian)

[2] Balayev, N.N. Determining the correlation between the flight parameters of the aircraft to recover flight information // Informasiya Texnologiyaları Problemləri. - 2010. - No 2. - p. 103-108. (in Azerbaijani)

[3] Alguliyev R.M., Orujov G.G., Sabziev E.N. On one method of recovering lost flight information // Proceedings of the III international conference "System Identification and Control Problems", Moscow, January 28-30, 2004, - p. 348-352. (in Russian)

[4] Azizov P. A. Development of methods and algorithms for the operational processing of flight data: Dissertation for the degree of Candidate of Technical Sciences. Institute of Information Technologies of ANAS, Baku, 2004. - 160 p. (in Russian)

[5] Alguliyev R.M., Orujov G.G., Sabziev E.N. Fusion of measurements to identify the flight path of an aircraft // Mekhatronika, Avtomatizatsiya, Upravleniye, No. 2, 2012, - p. 57-60, (Moscow). (in Russian)

[6] Bondarchuk I.E., Kharin V.I. Aviation and avionics equipment of the AN24 aircraft. - M .: Transport, 1975. - 280 p. (in Russian)

[7] Solovyev V.I., Shabalov P.G. Inertial navigation systems: A study guide / Samara: Publishing house of the Samara State Aerospace University, 2011. - 72 p. (in Russian)

[8] McCracken D., Dorn W. Numerical Methods and Fortran Programming. - John Wiley, New York, 1967. - 457 p. 